the field-effect device that was the basis for most of his claims. Surprisingly, there were also no photographs of the various characters in the story. And because of the structure of the book, the actual chronology of events was slightly difficult to follow. I also feel that many readers will take exception to how leniently Schön's senior coauthors were dealt with in the book, perhaps because they were willing to be interviewed (those who refused were treated more harshly). In fact, a serious discourse of the responsibilities of senior authors, management, journal editors and referees in the scientific process would have been a welcome addition.

For those who are looking for more details than those offered here, the official investigation, the 'Beasley Report', is highly recommended reading. I can still remember the state of shock I felt when reading it. Despite these minor reservations, I wholeheartedly recommend reading Reich's sobering account of how science can go wrong. In particular, this book, along with Feynman's 'cargo cult' address, would be wonderful components to any class dealing with ethics in science.

REVIEWED BY MICHAEL R. NORMAN Michael R. Norman, who is thankful that his name was not mentioned in this book, is in the Materials Science Division, Argonne National Laboratory, 9700 S Cass Avenue, Argonne, Illinois 60439, USA. e-mail:norman@anl.gov

\title{
Gravitation for the nation
}
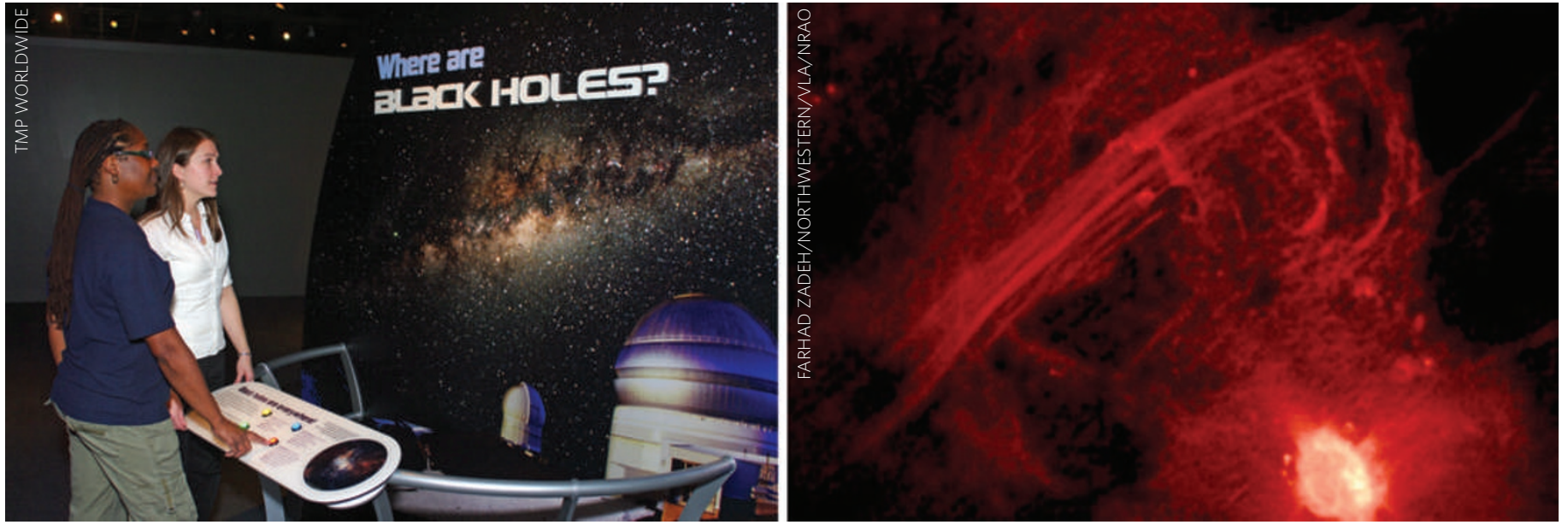

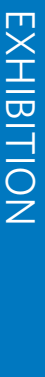

If their starring roles in films, pop songs and video games are anything to go by, black holes are deeply entrenched in the popular psyche. A Google search on black holes produces nearly 26 million hits, compared with 47 million for astronomy, 21 million for stem cells and 7.8 million for string theory. Beyond just good marketing (would black holes have become so popular had they been called 'gravitationally completely collapsed stars'?), there is something inherently mysterious and powerful about them. Something dangerous. And we can't see them: they're black against a (mostly) black background. So how do we know they are even there? A new exhibit at the Museum of Science in Boston, Black Holes: Space Warps \& Time Twists, aims to educate people about black holes.

A black hole is black because nothing, not even light, can escape its immense gravitational field. Once matter passes the 'event horizon' - a spacetime membrane within which all the paths that a particle may take are warped back further into the black hole - it cannot escape. However, there are indirect signs of a black hole's presence. Gravitational lensing warps light from a background source. Also, black holes are messy eaters. As they consume interstellar dust or matter from a companion star, the spiralling-in matter heats up and gives off radiation. The birth of a black hole can emit intense radiation as well, whether it's from the collision of two neutron stars or the collapse of a massive star.

Such properties of black holes, plus the convoluted history of their discovery, are explored at the 16 stations of the exhibit, with scientists and teenaged 'black-hole explorers' standing by. Even common misconceptions are dispelled. For example, many believe that black holes voraciously suck up everything within a vast volume in fact they don't, you have to get quite close. The highlight of the exhibit is a journey to a black hole in an 'immersive excursion pod' named The Singularity.
According to Mary Dussault, one of the designers, it "is a booth that contains a touch-screen control panel and a large plasma screen that gives the visitor the feel that they are in a 'pod' as they use the touch screen to control their virtual trip to a black hole". On the edge of the black hole lies the wreck of a spacecraft, from which it's possible to retrieve a treasure. There are additional opportunities to experience the effect of the black hole on the environment: warped space, time dilation and strong magnetic fields.

The exhibit runs until 7 September 2009. Given that many of the interactive parts of the stations were designed in collaboration with teenagers (the hardest group to please) people of all ages should enjoy themselves. To complement this exhibit, the museum's planetarium has a new show, Journey to the Edge of Space and Time, which traces the history of our quest to understand the Universe.

MAY CHIAO 